\title{
Factors Affecting Hospital Length of Stay in Patient with Diabetic Foot Ulcer
}

\author{
Patrianef Darwis $^{1}$, Bakti H Simanjuntak ${ }^{2}$, Grace Wangge ${ }^{3}$, Deddy Pratama ${ }^{4}$, Ahmad Bakri $^{5}$, \\ Rizky Saputra Telaumbanua ${ }^{6}$
1,2,4,5,6 Vascular and Endovascular Division, Department of Surgery, Faculty of Medicine Universitas Indonesia / Cipto Mangunkusumo General Hospital
${ }^{3}$ Departement of Community Medicine, Faculty of Medicine Universitas Indonesia

\begin{abstract}
Background. Foot ulcer is one of the most common complications in diabetes mellitus patients. This condition prolongs hospital length of stay (LOS) and increases hospitalization cost. This study aims to assess factors that affect the LOS in patients with the mentioned condition.

Methods. This is a retrospective cohort study of diabetes mellitus patients with foot ulcer who were hospitalized in Cipto Mangunkusumo General Hospital from January 2015 to April 2016. There were 120 patients recruited and then divided into two groups according to their hospitalization duration, which was short and long. Univariate analysis was conducted in predicted factors including gender, ankle-brachial index, ulcer size, ulcer depth, leukocyte count, treatment, cardiovascular comorbidity, blood pressure, smoking history, septicemia, ketoacidosis, hypoalbuminemia, and upper respiratory tract infection. Chi-Square tests were performed to analyze the association of those factors with LOS. The odds ratio of each variable was evaluated using logistic regression analysis.
\end{abstract}

Result. In this study, the mean of LOS was 26 days ( $2-87$ days). Factors that significantly correlated with LOS were ankle-brachial index ( $p$ 0.041, OR 2.275, CI $95 \% 1.025$ - 5.041), ulcer size ( $p$ 0.044, OR 3.038, CI $95 \% 1.032$ - 9.942), smoking history ( $p 0.022$, OR 2.434, CI $95 \%$ 1.125 - 5.265), sepsis ( $p<0.001$, OR 4.240, CI $95 \% 1.908-9.423)$, and ketoacidosis $(p<0.001$, OR 8.611 , CI $95 \% 3.396$ - 21.835) In multivariate analysis, the most significant factor was ketoacidosis ( $<<0.001$, OR 8.360, CI $95 \% 3.209-21.780)$.

Conclusion. Ketoacidosis is the most significant factor that prolonged hospital stays in a patient with diabetic foot ulcer.

Keywords: Diabetic foot ulcer, Length of stay 


\section{Introduction}

Diabetes mellitus is one of the world's most common health burdens. The global prevalence in 2019 was estimated by 9.3\% (463 million people). ${ }^{1}$ Meanwhile in Indonesia, the disease's prevalence was $8.5 \%$ according to National Basic Health Research (RISKESDAS) 2018. This disease causes various complications and one of them is diabetic foot ulcer. The lifetime risk of diabetic foot ulcer in diabetic patient ranges from $15-20 \%{ }^{3}$ Most patients with diabetic foot ulcer will require hospitalization with various duration. A study at a Tertiary Hospital in Semarang, Indonesia, revealed that the mean length of stay (LOS) for patient with a diabetic foot ulcer was 17.8 days $(5-71$ days). ${ }^{4} \mathrm{~A}$ wide gap in LOS is determined by various factors. Therefore, this study aims to reveal those factors that affecting LOS in patient with diabetic foot ulcer. Understanding those factors might play an important role in improving strategies in managing patients with diabetic foot ulcer.

\section{Methods}

This was a single-centered, retrospective cohort study that was conducted at Cipto Mangunkusumo General Hospital (RSCM) in Jakarta, Indonesia. This study was approved by the Ethics Committee of Faculty of Medicine, University of Indonesia. Data were collected from medical record of patients both men and women with diabetic foot ulcer who were hospitalized at RSCM from January 2015 to April 2016. Patients who had died during treatment or underwent major amputation were exclude. We observed 12 independent variables among a total of 120 patients that were recruited using consecutive sampling.

Patients in this study were classified into two groups according to their LOS, which were short (below mean duration) and long (above or equal to mean duration). 
Data about factors that potentially affect LOS (independent variables) were collected. Those independent variables were (1) gender, (2) ankle-brachial index (ABI), (3) ulcer size, (4) ulcer depth, (5) leukocyte count, (6) treatment, (7) cardiovascular morbidity, (8) blood pressure, (9) smoking history, (10) septicemia (according SIRS criteria), (11) ketoacidosis (blood glucose $>250 \mathrm{mg} / \mathrm{dl}$, acidosis metabolic, ketonuria and/or ketonemia), and (12) hypoalbuminemia.

Analysis methods that were conducted in this study were univariate, bivariate, and multivariate analysis. Univariate analysis was performed on each independent variable to find a descriptive figure of each variable. Bivariate analysis with Chi-Square test was conducted to find the association of each independent variable with LOS. Finally, multivariate analysis using logistic regression model was conducted to know the odds ratio (OR) of each independent variable. A p-value $<0.05$ was considered significant. The analysis was carried out using SPSS 20.0 for Windows.

\section{Results}

Table 1 shows the result of observed variables from the subjects. LOS varied from $2-87$ days, with the mean duration of 26 days. Sixty seven patients were categorized as patients with short hospitalization duration and 53 patients as patients with long hospitalization duration. The distribution of men and women were relatively equal, with $53.33 \%$ and $46.67 \%$ respectively. Most patients had normal ABI (70.00\%), small or below $79 \mathrm{~cm}$ wound size (77.50\%), Wagner's grade 2 ulcer depth (77.50\%), and high leukocyte count $(96.70 \%)$. According to the treatment record, most patients were only treated with debridement $(62.50 \%)$. Most patients did not have cardiovascular comorbidity (81.67\%), smoking history (65.83\%), septicemia $(65.00 \%)$, or acute respiratory infection (90.00\%). However, $62.50 \%$ of patients included had ketoacidosis and $80.83 \%$ had hypoalbuminemia.

In bivariate analysis as presented in table 2, there were five factors that had statistically significant association with LOS, which were ABI $(p$ 0.041, OR 2.275, CI $95 \% 1.027$ 5.041), ulcer size ( $p$ 0.044, OR 3.038, CI $95 \%$ $1.032-9.942$ ), smoking history ( $p \quad 0.022$, OR 
2.434, CI $95 \% 1.125$ - 5.265), sepsis ( $p$ 0.000, OR 4.240 CI $95 \% 1.908$ - 9.423), and ketoacidosis $(p<0.001$, OR 8.611, CI $95 \%$ $3.396-21.835)$.

Independent variables that had p-value $<0.25$ in the bivariate analysis were then included in the logistic regression test. The mentioned variables were ABI, ulcer size, leukocyte count, treatment, smoking history, sepsis, ketoacidosis, and upper respiratory tract infection. By using logistic regression test on those variables, we found three variables that had the most impact on LOS, which were ketoacidosis, $\mathrm{ABI}$, and smoking history (table 3). However, ketoacidosis was the only factor that had statistically significant correlation with LOS $(p<0.001$, OR 8.360, CI $95 \% 3.209-$ 21.780).

\section{Discussion}

At present, there are very few studies that examine the factors affecting the LOS of patients with diabetic foot ulcer. The latest research is a study conducted by Choi et al. in 2017. That study was a retrospective study conducted among 164 patients at Konkuk University Chungju Hospital in South Korea.
The examined factors were sex, age, duration of diabetes, smoking status, body mass index, underlying comorbidities (hypertension and diabetic nephropathy), wound characteristics, number of wound, severity, type of surgery, leukocyte count, CRP levels, ESR, albumin, protein, HbAlc, and 7-days mean blood glucose level. In that study, factors that associate with LOS is wound severity (OR 1.423), leukocyte count (OR 1.423), C-reactive protein (OR 1.079), albumin (OR 0.263) and blood glucose (OR 1.018). ${ }^{5}$

As for our study, several new factors compared to previous studies have been found to have association with LOS. Those factors include ABI, ulcer size, smoking history, sepsis, and ketoacidosis which have statistically significant association in bivariate analysis. However, after conducting multivariate analysis, ketoacidosis has been found to be the only independent predictor $(p<0.001$, OR 8.360, CI $95 \% 3.209$ $-21.780)$. Other factors in our study such as gender, ulcer depth, leukocyte count, treatment strategies, cardiovascular morbidity, blood pressure, hypoalbuminemia, and upper respiratory infection do not have significant correlation with LOS. 
Among a total of 120 patients included in this study, the distribution of subjects was 53\% men and $47 \%$ women. Although gender shows no significant result in this study, this study descriptively shows that the number of diabetic foot ulcer in men and women at RSCM are relatively the same.

$\mathrm{ABI}$ is the ratio between the systolic blood pressure of the lower extremity, specifically the ankle, divided by the highest systolic in the upper extremity. This is a non-invasive method to screen peripheral arterial disease (PAD). ${ }^{6,7}$ Lower ABI indicates problems with blood flow, while adequate blood flow is needed in optimal wound healing. Therefore, patients with lower ABI will have disrupted wound healing process. Olivieri B et al. in 2018 stated that wound healing in patients with peripheral arterial disease often stops at one particular stage, most often at the inflammatory stage. ${ }^{8}$ This will ultimately prolong the hospitalization duration. Furthermore, our bivariate analysis shows that there is a correlation between $\mathrm{ABI}$ and LOS in chronic wounds, specifically diabetic foot ulcer ( $p 0.041$, OR 2.275, CI $95 \%$ $1.027-5.041)$.
The influence of ulcer size on LOS has also been found to be one of the significant results on this study. Previous studies had examined the relationship between ulcer size and healing time but did not examine the ulcer size relationship with LOS. One such study is by Zimny et al. in 2004, in which they found that the greater the ulcer sizes the faster the wound radius reduction. However, when they examined it with healing time, the relationship was linear, as larger ulcer size will need a longer healing time..$^{9,10}$ In our study, ulcer size were classified into three categories, which are small $(<79 \mathrm{~cm})$, medium $(79-141 \mathrm{~cm})$, and large $(141 \mathrm{~cm})$. We found that ulcer size has statistically significant association with LOS ( $p$ 0.044, OR 3.038, CI $95 \% 1.032$ - 9.942).

Sepsis is described as abnormal body's systemic immunological responses to infection. This is a medical emergency that can lead to end-stage organ dysfunction and death. ${ }^{11,12}$ Diabetes patients are prone to infection and sepsis due to immune deficiency with chronic inflammation and immune suppression. ${ }^{13}$ Center for Disease Control and Prevention (CDC) reported that patients hospitalized with septicemia had $75 \%$ longer LOS than patients hospitalized without septicemia. ${ }^{14}$ However, 
currently there is no study that specifically correlate sepsis with LOS in diabetic foot ulcer. This study confirms the effect of sepsis on LOS including and specifically in patient with diabetic foot ulcer $(p<0.001$, OR 4.240 CI 95 $\% 1.908$ - 9.423). However, in the multivariate analysis, this finding was not statistically significant.

In our multivariate analysis with logistic regression test, it was found that the independent variable that remained consistently significant as a factor affecting the LOS is ketoacidosis $(p<0.001$ OR 8.360, CI $95 \%$ 3.209 - 21.780). Ketoacidosis is an acute and serious complication in diabetes. This is a metabolic emergency state consists of biochemical triad of hyperglycemia, ketonemia, and high anion gap. ${ }^{15,16}$ Currently there is no study examining the relationship between ketoacidosis and LOS in patient with diabetic foot ulcer. Study that still bears some resemblance is Choi et al.'s study, wherein that study they found that high blood glucose level prolonged LOS. However, this study has an important difference, in which the cut-off value that was used was the subjects mean blood glucose level which was $218 \mathrm{mg} / \mathrm{dL} .^{5}$ Therefore, this study does not represent ketoacidosis, as the cut-off value of blood glucose level in ketoacidosis diagnostic criteria are $>250 \mathrm{mg} / \mathrm{dL} .{ }^{17}$ Moreover, ketoacidosis is not limited in hyperglycemia, but also includes ketonemia and high anion gap. ${ }^{15,16}$

There are several reasons why patient with ketoacidosis need longer treatment. First, because patients with ketoacidosis experience severe blood glucose dysregulation, whereas controlled blood glucose is an indicator of a patient can be discharged. Additionally, Melissa et al. has reported that the median LOS in diabetic ketoacidosis patient was 3 days. ${ }^{18}$ Second, most diabetic foot ulcer patient in this study have higher infection level (116 out 120 patients have high leukocyte count). Infection itself is one of the most common triggers of ketoacidosis. ${ }^{19}$ Combination of ketoacidosis and infection will probably result in prolonged LOS because controlled infection is also one of the discharge indicators in patient with diabetic foot ulcer.

\section{Conclusion}

In conclusion, in this study we found that ABI, ulcer size, smoking history, sepsis, and ketoacidosis are factors that have significant 
association with LOS in patient with diabetic foot ulcer. Among them, ketoacidosis was found to be the most significant factor. This is quite a new finding among studies of diabetic foot ulcer. However, we acknowledged, as a limitation in this study, that the medical record data was incomplete thus making it difficult for us to explore other factors. Other studies with bigger sample size and more factors needs to be conducted in order to make a more comprehensive scoring system to predict LOS in patient with diabetic foot ulcer.

(ISSN:0216-0951 J Bedah Indonesia. 2019; 47:2)

\section{References}

1. Saeedi P, Petersohn I, Salpea P, Malanda B, Karuranga S, Unwin $\mathrm{N}$ et al. Global and regional diabetes prevalence estimates for 2019 and projections for 2030 and 2045: Results from the International Diabetes Federation Diabetes Atlas, 9th edition. Diabetes Research and Clinical Practice. 2019;157:107843.

2. Kementerian Kesehatan Republik Indonesia. Hasil utama riskesdas 2018.
Jakarta: Badan Penelitian dan Pengembangan Kesehatan; 2018

3. Chun D, Kim S, Kim J, Yang H, Kim J, Cho $\mathrm{J}$ et al. Epidemiology and Burden of Diabetic Foot Ulcer and Peripheral Arterial Disease in Korea. Journal of Clinical Medicine. 2019;8(5):748.

4. Pemayun T, Naibaho R. Clinical profile and outcome of diabetic foot ulcer, a view from tertiary care hospital in Semarang, Indonesia. Diabetic Foot \& Ankle. 2017;8(1):1312974.

5. Choi S, Kim C, Jo D, Lee M, Kim J, Choi $\mathrm{H}$ et al. Factors Associated with a Prolonged Length of stay in Patients with Diabetic Foot: A Single-Center Retrospective Study. Archives of Plastic Surgery. 2017;44(6):539-544.

6. Pratama D, Ibrahim H, Suhartono R, Patrianef et al. Panduan Praktik Klinis RSCM: Lower extremity peripheral arterial disease. Jakarta: RSCM; 2019

7. McClary K, Massey P. Ankle Brachial Index (ABI) [Internet]. Ncbi.nlm.nih.gov. 2020 [cited 21 April 2020]. Available from: https://www.ncbi.nlm.nih.gov/books/N BK544226/ 
8. Yates T, Vianna S, Adenikinju $\mathrm{O}$, Beasley R, Houseworth J, Olivieri B. On the Cutting Edge: Wound Care for the Endovascular Specialist. Seminars in Interventional Radiology. 2018;35(05):406-426.

9. Zimny S, Schatz H, Pfohl M. The Effects of Ulcer Size on the Wound Radius Reductions and Healing Times in Neuropathic Diabetic Foot Ulcers. Experimental and Clinical Endocrinology \& Diabetes. 2004;112(04):191-194.

10. Zimny S, Pfohl M. Healing Times and Prediction of Wound Healing in Neuropathic Diabetic Foot Ulcers: a Prospective Study. Experimental and Clinical Endocrinology \& Diabetes. 2005;113(02):90-93.

11. Berg D, Gerlach H. Recent advances in understanding and managing sepsis. F1000Research. 2018;7:1570.

12. Gyawali B, Ramakrishna K, Dhamoon A. Sepsis: The evolution in definition, pathophysiology, and management. SAGE Open Medicine. 2019;7:205031211983504.

13. Frydrych L, Fattahi F, He K, Ward P, Delano M. Diabetes and Sepsis: Risk,
Recurrence, and Ruination. Frontiers in Endocrinology. 2017;8.

14. Hall MJ, Williams SN, Defrances SJ, Golosinskiy A. Inpatient care for septicemia or sepsis: a challenge for patients and hospitals. NCHS Data Brief. 2011;6(62):1-8

Ghimire P, Dhamoon A. Ketoacidosis [Internet]. Ncbi.nlm.nih.gov. 2020

[cited 26 April 2020]. Available from: https://www.ncbi.nlm.nih.gov/books/N BK534848/

15. Gosmanov A, Gosmanova E, Kitabchi A. Hyperglycemic Crises: Diabetic Ketoacidosis (DKA), And Hyperglycemic Hyperosmolar State (HHS) [Internet]. Ncbi.nlm.nih.gov. 2020 [cited 26 April 2020]. Available from:

https://www.ncbi.nlm.nih.gov/books/N BK279052/

16. Table 1, Criteria and classification of (DKA) - Endotext - NCBI Bookshelf [Internet]. Ncbi.nlm.nih.gov. 2020 [cited 26 April 2020]. Available from: https://www.ncbi.nlm.nih.gov/books/N BK279146/table/diabketoacidosis.table1crit/ 
17. Lee M, Calder G, Santamaria J, MacIsaac R. Diabetic ketoacidosis in adult patients: an audit of factors

influencing time to normalisation of metabolic parameters. Internal

Medicine Journal. 2018;48(5):529-53 


\section{Tables}

Table 1. Characteristic of the subjects

\begin{tabular}{|c|c|c|}
\hline Variable & Total (n) & Percentage (\%) \\
\hline \multicolumn{3}{|l|}{ Gender } \\
\hline Men & 64 & 53.33 \\
\hline Women & 56 & 46.67 \\
\hline \multicolumn{3}{|l|}{ Ankle Brachial Index (ABI) } \\
\hline Normal $(0.9-1.4)$ & 84 & 70.00 \\
\hline Abnormal & 36 & 30.00 \\
\hline \multicolumn{3}{|l|}{ Ulcer Size } \\
\hline Small $(<79 \mathrm{~cm})$ & 93 & 77.50 \\
\hline Medium $(79-141 \mathrm{~cm})$ & 10 & 8.33 \\
\hline Large $(>141 \mathrm{~cm})$ & 17 & 14.17 \\
\hline \multicolumn{3}{|c|}{ Ulcer depth (Wagner Classification) } \\
\hline Grade 1 & 3 & 2.50 \\
\hline Grade 2 & 93 & 77.50 \\
\hline Grade 3 & 15 & 12.50 \\
\hline Grade 4 & 9 & 7.50 \\
\hline \multicolumn{3}{|l|}{ Leukocyte } \\
\hline Normal & 4 & 3.30 \\
\hline High $(>10.000)$ & 116 & 96.70 \\
\hline \multicolumn{3}{|l|}{ Treatment } \\
\hline Debridement & 75 & 62.50 \\
\hline Debridement + Amputation & 37 & 30.83 \\
\hline Debridemen + Revascularization & 8 & 6.67 \\
\hline
\end{tabular}




\section{Cardiovascular comorbidity}

Yes

22

18.33

No

98

81.67

\section{Blood pressure}

Hypertension

67.50

Normal

81

32.50

Smoking history

Yes

41

34.17

No

79

65.83

Sepsis

Yes

42

35.00

No

78

65.00

Ketoacidosis

Yes

No

\section{Hypoalbuminemia}

Yes

No

Acute respiratory infection

Yes

12

10.00

No

108

90.00

\section{Length of stay}

\begin{tabular}{lrr} 
Short $(\leq 26$ days $)$ & 67 & 55.83 \\
Long $(>26$ days $)$ & 53 & 44.16 \\
\hline Total & 120 & 100 \\
\hline
\end{tabular}


Table 2. Correlation between independent variable and LOS

\begin{tabular}{|c|c|c|c|c|c|}
\hline \multirow{2}{*}{ Variable } & \multicolumn{2}{|c|}{ Length of stay (\%) } & \multirow{2}{*}{$p$} & \multirow{2}{*}{$\begin{array}{c}\text { OR } \\
\text { (crude } \\
\text { ) }\end{array}$} & \multirow{2}{*}{ CI 95\% } \\
\hline & Short & Long & & & \\
\hline \multicolumn{6}{|l|}{ ABI } \\
\hline Abnormal & $15(41.7)$ & $21(58.3)$ & 0.041 & 2.275 & $1.027-5.041$ \\
\hline Normal & $52(61.9)$ & $32(38.1)$ & & & \\
\hline \multicolumn{6}{|l|}{ Ulcer size } \\
\hline Small & $58(62.4)$ & $35(37.6)$ & 1 & 1 & \\
\hline Medium & $3(30)$ & $7(70)$ & 0.061 & 3.867 & 0.938 \\
\hline Large & $6(35.3)$ & $11(64.7)$ & 0.044 & 3.038 & 15.934 \\
\hline
\end{tabular}

\section{Wagner Classification}

Grade 1

Grade 2

Grade 3

Grade 4

$$
\begin{array}{rrrrr}
2(66.7) & 1(33.3) & 1 & 1 & 1 \\
52(55.9) & 41(44.1) & 0.714 & 1.577 & 0.138- \\
6(40) & 9(60) & 0.410 & 3 & 18.004 \\
7(77.8) & 2(22.2) & 0.702 & 0.571 & 0.220- \\
& & & & 40.931 \\
& & & & 0.032- \\
& & & & 10.069
\end{array}
$$

\section{Leukocyte}

$\begin{array}{lrrrrr}\text { High }(>10.000) & 54(53.5) & 47(46.5) & 0.228 & 0.530 & 0.187-1.505 \\ \text { Normal } & 13(68.4) & 6(31.6) & & & \\ \text { Treatment } & & & & & \\ \text { Debridement } & 47(62.7) & 28(37.3) & 1 & 1 & 1 \\ \text { Debrid+amputation* } & 18(48.6) & 19(51.4) & 0.159 & 1.772 & 0.799-3.930 \\ & 2(25) & 6(75) & 0.057 & 5.036 & 0.951- \\ & & & & & 26.679\end{array}$


Debrid+revascularization

\section{Cardiovascular}

comorbidity

$$
10(45.5) \quad 0.893 \quad 1.066 \quad 0.421-2.699
$$

Yes

$12(54.5) \quad 43(43.9)$

No

$55(56.1)$

Blood pressure

Hypertension

$24(61.5) \quad 15(38.5)$

0.383

$1.414 \quad 0.646-3.081$

Normal

43 (53.1) $38(46.9)$

\section{Smoking history}

Yes

No

$17(41.5) \quad 24(58.5)$

0.022

$50(63.3) \quad 29(36.7)$

Sepsis

Yes

No

$14(33.3) \quad 28(66.7)$

0.000

4.240

$1.908-9.423$

$53(67.9) \quad 25(32.1)$

Ketoacidosis

Yes

No

Hypoalbuminemia

Yes

No

$29(38.7) \quad 46(61.3)$

0.000 8.611 $3.396-$

$38(84.4) \quad 7(15.6)$ 21.835

Acute respiratory tract

\section{infection}

$\begin{array}{lrrrrr}\text { Yes } & 4(33.3) & 8(66.7) & 0.098 & 2.800 & 0.794-9.868 \\ \text { No } & 63(58.3) & 45(41.7) & & & \end{array}$

*debridement 
Table 3. Multivariate analysis

\begin{tabular}{lrrr}
\hline Variable & Adjusted OR & $\boldsymbol{p}$-value & CI 95\% \\
\hline ABI & 0.473 & 0.103 & $0.193-1.164$ \\
Ketoacidosis & 8.360 & $\mathbf{0 . 0 0 0}$ & $3.209-21.780$ \\
Smoking history & 2.168 & 0.080 & $0.911-5.161$ \\
\hline
\end{tabular}

